\title{
Commentary on "The Urine Microbiome of Healthy Men and Women Differs by Urine Collection Method"
}

\author{
Seung-Ju Lee \\ Department of Urology, St. Vincent's Hospital, The Catholic University of Korea, Suwon, Korea
}

To the editor,

I read with great interest the article titled "The Urine Microbiome of Healthy Men and Women Differs by Urine Collection Method" published by Pohl et al. in the International Neurourology Journal in March 2020 [1]. The authors concluded that the urinary microbiome differed according to urine collection methods and that sex differences in the core microbiome exist.

When I analyzed articles that studied urinary microbiome in healthy individuals, or healthy controls in case-control studies, most studies showed similar results (Table 1). Lactobacillus was the predominant genus in the urinary microbiome in females, whereas a more heterogeneous group of microbiomes was shown in males [2-23]. The study by Wolfe et al. [5] was the only study that compared different urine collection methods, and they concluded that the best methods are suprapubic aspiration and transurethral catheterization.

There are still clear limitations in the study of the human urinary microbiome. Due to the heterogeneous design of urinary microbiome studies, it is difficult to make comparisons between studies and to draw conclusions. There are differences according to the urine collection method, 16s rRNA analysis tools, statistical methods, and the taxonomic database on which the reporting of genera or species is based. These heterogeneous study designs cause discrepancies in results. In addition, as the authors clearly pointed out, many other factors can influence the urinary microbiome, such as age, sex, dietary habits, infections and antimicrobial use, hormonal status, and regional variation.

Several points should be considered in future studies involv- ing the urinary microbiome. First, a larger sample size is needed. Second, standardization of methodology and reporting is necessary to facilitate comparisons between studies. Third, more studies on the normal urinary microbiome and factors influencing its composition are needed. Age, sex, and urine collection methods are known factors, but many other factors likely exist. One point to consider in this regard is that it may be impossible to define a "universal norm" regarding the urinary microbiome. Rather than defining a universal norm, identifying the normal urinary microbiome in each individual and using it as a personalized reference for future disease may be a more reasonable approach. Fourth, the connection between dysbiosis and disease should be more clearly identified. When this connection is found, it may become possible to use the urinary microbiome in diagnosis or treatment. Lastly, studies have suggested that the overall composition and richness of the microbiota play an important role in modulating vaccine response [24]. In this regard, the effects of the urinary microbiome on vaccinations for urinary tract infection may be an interesting topic for future studies.

- Conflict of Interest: No potential conflict of interest relevant to this article was reported.

\section{REFERENCES}

1. Pohl HG, Groah SL, Perez-Losada M, Ljungberg I, Sprague BM, Chandal N, et al. The urine microbiome of healthy men and women differs by urine collection method. Int Neurourol J 2020;24:41-51.

Corresponding author: Seung-Ju Lee (iD https://orcid.org/0000-0003-0072-8010 Department of Urology, St. Vincent's Hospital, The Catholic University of Korea, 93 Jungbu-daero, Paldal-gu, Suwon 16247, Korea

E-mail: lee.seungju@gmail.com

Submitted: May 14, 2020 / Accepted after revision: May 19, 2020

(c) (1) 5 This is an Open Access article distributed under the terms of the Creative Commons Attribution Non-Commercial License (https://creativecommons.org/licenses/by-nc/4.0/) which permits unrestricted non-commercial use, distribution, and reproduction in any medium, provided the original work is properly cited. 
Table 1. Articles that studied the urinary microbiome in healthy individuals, or healthy controls in case-control studies

\begin{tabular}{|c|c|c|c|c|c|}
\hline Study & No. & Sex & Age & $\begin{array}{l}\text { Urine } \\
\text { collection }\end{array}$ & Microbiome \\
\hline $\begin{array}{l}\text { Siddiqui et al. } \\
\text { [2] (2011) }\end{array}$ & 8 & $\mathrm{~F}$ & $27-67 \mathrm{yr}$ & $\mathrm{CCU}$ & $\begin{array}{l}\text { Lactobacillus, Prevotella, Gardnerella, Peptoniphilus, Dialister, Finegoldia, } \\
\text { Anaerococcus, Allisonella, Streptococcus, Staphylococcus }\end{array}$ \\
\hline $\begin{array}{l}\text { Wolfe et al. } \\
\text { [5] (2012) }\end{array}$ & 12 & $\mathrm{~F}$ & NA & $\begin{array}{l}\text { CCU, } \\
\text { TUC, } \\
\text { SPA }\end{array}$ & $\begin{array}{l}\text { Lactobacillus, Actinobaculum, Aerococcus, Anaerococcus, Atopobium, } \\
\text { Burkholderia, Corynebacterium, Gardnerella, Prevotella, Ralstonia, Sneathia, } \\
\text { Staphylococcus, Streptococcus, Veillonella }\end{array}$ \\
\hline $\begin{array}{l}\text { Fouts et al. } \\
\text { [3] (2012) }\end{array}$ & 11 & $\begin{array}{l}\mathrm{M} \\
\mathrm{F}\end{array}$ & $\begin{array}{l}24-50 \mathrm{yr} \\
22-51 \mathrm{yr}\end{array}$ & MSU & $\begin{array}{l}\text { Corynebacterium, Staphylococcus, Streptococcus, Lactobacillus, Gardnerella, } \\
\text { Veillonella } \\
\text { Lactobacillus, Corynebacterium, Staphylococcus, Streptococcus, Prevotella }\end{array}$ \\
\hline $\begin{array}{l}\text { Nelson et al. } \\
\text { [4] (2012) }\end{array}$ & 18 & M & $14-17 \mathrm{yr}$ & FCU & $\begin{array}{l}\text { Corynebacterium, Lactobacillus, Staphylococcus, Gardnerella, Streptococcus, } \\
\text { Anaerococcus, Veillonella, Prevotella, Escherichia }\end{array}$ \\
\hline $\begin{array}{l}\text { Lewis et al. } \\
\text { [6] (2013) }\end{array}$ & $\begin{array}{r}6 \\
10\end{array}$ & $\begin{array}{l}\mathrm{M} \\
\mathrm{F}\end{array}$ & $\begin{array}{l}39-83 \mathrm{yr} \\
26-90 \mathrm{yr}\end{array}$ & $\mathrm{CCU}$ & $\begin{array}{l}\text { Firmicutes } \\
\text { Firmicutes, Actinobacteria, Bacteroidetes }\end{array}$ \\
\hline $\begin{array}{l}\text { Hilt et al. } \\
\text { [7] (2014) }\end{array}$ & 24 & $\mathrm{~F}$ & NA & TUC & $\begin{array}{l}\text { Lactobacillus, Corynebacterium, Streptococcus, Actinomyces, Staphylococcus, } \\
\text { Aerococcus, Gardnerella, Bifidobacterium, Actinobaculum }\end{array}$ \\
\hline $\begin{array}{l}\text { Pearce et al. } \\
\text { [8] (2014) }\end{array}$ & 58 & $\mathrm{~F}$ & $35-65 \mathrm{yr}$ & TUC & $\begin{array}{l}\text { Lactobacillus, Gardnerella, Corynebacterium, Enterobacteriaceae, Anaerococcus, } \\
\text { Bifidobacterium, Streptococcus, Staphylococcus, Sneathia, Peptoniphilus, } \\
\text { Atopobium, Rhodanobacter, Trueperella, Alloscardovia, Veillonella }\end{array}$ \\
\hline $\begin{array}{l}\text { Karstens et al. } \\
\text { [9] (2016) }\end{array}$ & 10 & $\mathrm{~F}$ & 58 (mean) & TUC & $\begin{array}{l}\text { Anoxybacillus, Lactobacillus, Prevotella, Gardnerella, Arthrobacter, Escherichia, } \\
\text { Shigella }\end{array}$ \\
\hline $\begin{array}{l}\text { Thomas-White et al. } \\
\text { [10] (2016) }\end{array}$ & 60 & $\mathrm{~F}$ & $35-65 \mathrm{yr}$ & TUC & $\begin{array}{l}\text { Lactobacillus, Gardnerella, Staphylococcus, Streptococcus, Enterococcus, } \\
\text { Bifidobacterium, Atopobium, Enterobacteriaceae }\end{array}$ \\
\hline $\begin{array}{l}\text { Wu et al. } \\
{[15](2017)}\end{array}$ & 25 & $\mathrm{~F}$ & 26 (mean) & TUC & $\begin{array}{l}\text { Lactobacillaceae, Prevotellaceae, Enterobacteriaceae, Veillonellaceae, } \\
\text { Tissierellaceae, Bifidobacteriales }\end{array}$ \\
\hline $\begin{array}{l}\text { Gottschick et al. } \\
{[12](2017)}\end{array}$ & 49 & $\mathrm{~F}$ & $19-62 \mathrm{yr}$ & MSU & Lactobacillus crispatus \\
\hline $\begin{array}{l}\text { Abernethy et al. } \\
\text { [11] (2017) }\end{array}$ & 20 & $\mathrm{~F}$ & $28-43 \mathrm{yr}$ & TUC & Lactobacillus acidophilus \\
\hline $\begin{array}{l}\text { Wang et al. } \\
\text { [14] (2017) }\end{array}$ & 21 & $\mathrm{~F}$ & 43 (mean) & MSU & Lactobacillus, Varibaculum, Porphyromonas, Prevotella, Bacteroides \\
\hline $\begin{array}{l}\text { Rani et al. } \\
\text { [13] (2017) }\end{array}$ & $\begin{array}{l}5 \\
3\end{array}$ & $\begin{array}{l}\mathrm{F} \\
\mathrm{M}\end{array}$ & $27-63 \mathrm{yr}$ & MSU & Proteobacteria, Firmicutes, Actinobacteria, Bacteroidetes \\
\hline $\begin{array}{l}\text { Wu et al. } \\
{[18](2018)}\end{array}$ & 18 & M & 55.5 (mean) & MSU & $\begin{array}{l}\text { Escherichia-Shigella, Staphylococcus, Streptococcus, Aeromonas, Acinetobacter, } \\
\text { Bacteroides, Lactobacillus }\end{array}$ \\
\hline $\begin{array}{l}\text { Komesu et al. } \\
\text { [17] (2018) }\end{array}$ & 84 & $\mathrm{~F}$ & 53 (mean) & TUC & Lactobacillus, Gardnerella, Tepidimonas, Prevotella \\
\hline $\begin{array}{l}\text { Bucevic Popovic et al. } \\
\text { [16] (2018) }\end{array}$ & 19 & M & $61-82 \mathrm{yr}$ & MSU & Firmicutes, Actinobacteria, Bacteroidetes, Proteobacteria \\
\hline $\begin{array}{l}\text { Meriwether et al. } \\
\text { [21] (2019) }\end{array}$ & 18 & $\mathrm{~F}$ & 33.7 (mean) & MSU & Lactobacillus, Prevotella \\
\hline $\begin{array}{l}\text { Bresler et al. } \\
\text { [19] (2019) }\end{array}$ & 20 & $\mathrm{~F}$ & 48 (mean) & MSU & Lactobacillus \\
\hline $\begin{array}{l}\text { Kassiri et al. } \\
{[20](2019)}\end{array}$ & 10 & M & 3 mo- 8 yr & TUC & Staphylococcus, Varibaculum, Peptoniphilus, Actinobaculum \\
\hline $\begin{array}{l}\text { Xie et al. } \\
\text { [23] (2020) }\end{array}$ & 21 & M & 44.2 (mean) & NA & Acinetobacter, Prevotella, Oscillospira, Parabacteroides, Fusobacterium \\
\hline $\begin{array}{l}\text { Liu et al. } \\
{[22](2020)}\end{array}$ & $\begin{array}{l}9 \\
3\end{array}$ & $\begin{array}{c}\mathrm{M} \\
\mathrm{F}\end{array}$ & 58.9 (mean) & TUC & Gardnerella, Pontibacter, Sphingomonas, Prevotella, Propionibacterium \\
\hline
\end{tabular}

CCU, clean catch urine; TUC, transurethral catheter; SPA, suprapubic aspirate; MSU, midstream urine; FCU, first catch urine; NA, not available. 
2. Siddiqui H, Nederbragt AJ, Lagesen K, Jeansson SL, Jakobsen KS. Assessing diversity of the female urine microbiota by high throughput sequencing of 16S rDNA amplicons. BMC Microbiol 2011;11:244.

3. Fouts DE, Pieper R, Szpakowski S, Pohl H, Knoblach S, Suh MJ, et al. Integrated next-generation sequencing of $16 \mathrm{~S} \mathrm{rDNA}$ and metaproteomics differentiate the healthy urine microbiome from asymptomatic bacteriuria in neuropathic bladder associated with spinal cord injury. J Transl Med 2012;10:174.

4. Nelson DE, Dong Q, Van der Pol B, Toh E, Fan B, Katz BP, et al. Bacterial communities of the coronal sulcus and distal urethra of adolescent males. PLoS One 2012;7:e36298.

5. Wolfe AJ, Toh E, Shibata N, Rong R, Kenton K, Fitzgerald M, et al. Evidence of uncultivated bacteria in the adult female bladder. J Clin Microbiol 2012;50:1376-83.

6. Lewis DA, Brown R, Williams J, White P, Jacobson SK, Marchesi JR, et al. The human urinary microbiome; bacterial DNA in voided urine of asymptomatic adults. Front Cell Infect Microbiol 2013;3:41.

7. Hilt EE, McKinley K, Pearce MM, Rosenfeld AB, Zilliox MJ, Mueller ER, et al. Urine is not sterile: use of enhanced urine culture techniques to detect resident bacterial flora in the adult female bladder. J Clin Microbiol 2014;52:871-6.

8. Pearce MM, Hilt EE, Rosenfeld AB, Zilliox MJ, Thomas-White K, Fok $\mathrm{C}$, et al. The female urinary microbiome: a comparison of women with and without urgency urinary incontinence. mBio 2014; 5:e01283-14.

9. Karstens L, Asquith M, Davin S, Stauffer P, Fair D, Gregory WT, et al. Does the urinary microbiome play a role in urgency urinary incontinence and its severity? Front Cell Infect Microbiol 2016;6:78.

10. Thomas-White KJ, Hilt EE, Fok C, Pearce MM, Mueller ER, Kliethermes S, et al. Incontinence medication response relates to the female urinary microbiota. Int Urogynecol J 2016;27:723-33.

11. Abernethy MG, Rosenfeld A, White JR, Mueller MG, LewickyGaupp C, Kenton K. Urinary microbiome and cytokine levels in women with interstitial cystitis. Obstet Gynecol 2017;129:500-6.

12. Gottschick C, Deng ZL, Vital M, Masur C, Abels C, Pieper DH, et al. The urinary microbiota of men and women and its changes in women during bacterial vaginosis and antibiotic treatment. Microbiome 2017;5:99.

13. Rani A, Ranjan R, McGee HS, Andropolis KE, Panchal DV, Hajjiri $Z$, et al. Urinary microbiome of kidney transplant patients reveals dysbiosis with potential for antibiotic resistance. Transl Res 2017; 181:59-70.

14. Wang H, Altemus J, Niazi F, Green H, Calhoun BC, Sturgis C, et al. Breast tissue, oral and urinary microbiomes in breast cancer. Oncotarget 2017;8:88122-38.

15. Wu P, Chen Y, Zhao J, Zhang G, Chen J, Wang J, et al. Urinary microbiome and psychological factors in women with overactive bladder. Front Cell Infect Microbiol 2017;7:488.

16. Bucevic Popovic V, Situm M, Chow CT, Chan LS, Roje B, Terzic J. The urinary microbiome associated with bladder cancer. Sci Rep 2018;8:12157.

17. Komesu YM, Richter HE, Carper B, Dinwiddie DL, Lukacz ES, Siddiqui NY, et al. The urinary microbiome in women with mixed urinary incontinence compared to similarly aged controls. Int Urogynecol J 2018;29:1785-95.

18. Wu P, Zhang G, Zhao J, Chen J, Chen Y, Huang W, et al. Profiling the urinary microbiota in male patients with bladder cancer in China. Front Cell Infect Microbiol 2018;8:167.

19. Bresler L, Price TK, Hilt EE, Joyce C, Fitzgerald CM, Wolfe AJ. Female lower urinary tract microbiota do not associate with IC/PBS symptoms: a case-controlled study. Int Urogynecol J 2019;30:183542.

20. Kassiri B, Shrestha E, Kasprenski M, Antonescu C, Florea LD, Sfanos KS, et al. A Prospective study of the urinary and gastrointestinal microbiome in prepubertal males. Urology 2019;131:204-10.

21. Meriwether KV, Lei Z, Singh R, Gaskins J, Hobson DTG, Jala V. The vaginal and urinary microbiomes in premenopausal women with interstitial cystitis/bladder pain syndrome as compared to unaffected controls: a pilot cross-sectional study. Front Cell Infect Microbiol 2019;9:92.

22. Liu F, Zhang N, Jiang P, Zhai Q, Li C, Yu D, et al. Characteristics of the urinary microbiome in kidney stone patients with hypertension. J Transl Med 2020;18:130.

23. Xie J, Huang JS, Huang XJ, Peng JM, Yu Z, Yuan YQ, et al. Profiling the urinary microbiome in men with calcium-based kidney stones. BMC Microbiol 2020;20:41.

24. Ciabattini A, Olivieri R, Lazzeri E, Medaglini D. Role of the microbiota in the modulation of vaccine immune responses. Front Microbiol 2019;10:1305. 